\title{
Risk of Melanoma and Nonmelanoma Skin Cancer Among Patients With Inflammatory Bowel Disease
}

\author{
Millie D. Long ${ }^{\star}, \ddagger$, Christopher F. Martin ${ }^{*}, \neq$, Clare A. Pipkin $§$, Hans H. Herfarth ${ }^{\star}, \ddagger$, Robert S. \\ Sandler ${ }^{*} \neq$, and Michael D. Kappelman ${ }^{\|, \neq}$ \\ *Division of Gastroenterology and Hepatology, Department of Medicine, University of North \\ Carolina at Chapel Hill, Chapel Hill \\ "Division of Gastroenterology and Hepatology, Department of Pediatrics, University of North \\ Carolina at Chapel Hill, Chapel Hill \\ ¥Center for Gastrointestinal Biology and Disease, Chapel Hill \\ $\S$ Department of Dermatology, Duke University, Durham, North Carolina
}

\begin{abstract}
BACKGROUND \& AIMS—Patients with inflammatory bowel disease (IBD) are at risk for certain malignancies. We aimed to determine the risk of melanoma and nonmelanoma skin cancer (NMSC) in patients with IBD and how medications affect these risks.

METHODS-We performed retrospective cohort and nested case-control studies using administrative data from the LifeLink Health Plan Claims Database from 1997 to 2009. The cohort comprised 108,579 patients with IBD, and each was matched to 4 individuals without IBD. The risk of melanoma and NMSC was evaluated by incidence rate ratio (IRR) and by adjusted Cox proportional hazard ratio (HR) modeling. In nested case-control studies, patients with melanoma or NMSC were matched to 4 patients with IBD without melanoma or NMSC. Conditional logistic regression was used to determine associations between medications and both skin cancers.
\end{abstract}

RESULTS-In the cohort, IBD was associated with an increased incidence of melanoma (IRR, 1.29 ; 95\% confidence interval [CI], 1.09-1.53). Risk was greatest among individuals with Crohn's disease (IRR, 1.45; 95\% CI, 1.13-1.85; adjusted HR, 1.28; 95\% CI, 1.00-1.64). The incidence of NMSC also increased among patients with IBD (IRR, 1.46; 95\% CI, 1.40-1.53) and was greatest among those with CD (IRR, 1.64; 95\% CI, 1.54-1.74). In the nested case-control studies, therapy with biologics increased the risk of melanoma (odds ratio [OR], 1.88; 95\% CI, 1.08-3.29). Patients who had been treated with thiopurines had an increased risk of NMSC (OR, 1.85; 95\% CI, 1.66-2.05).

\footnotetext{
(C) 2012 by the AGA Institute

Address requests for reprints to: Millie D. Long MD, MPH, Campus Box 7080, University of North Carolina at Chapel Hill, Chapel Hill, North Carolina 27599-7080. millie_long@med.unc.edu; fax: (919) 966-6842.

Conflicts of interest

The authors disclose no conflicts.
} 
CONCLUSIONS-Immunosuppression increases the risk of melanoma and NMSC among patients with IBD. The risk of melanoma is increased by use of biologics, and the risk of NMSC is increased by use of thiopurines. Patients with IBD should be counseled and monitored for skin cancer.

\section{Keywords}

Malignancy; Cancer Risk; Epidemiology; Side Effect

It is estimated that 68,130 Americans were diagnosed with melanoma and 8700 Americans died of melanoma in 2010. The age-adjusted incidence rate for melanoma in the United States from 2004 to 2008 was 20.8 cases per 100,000 men and women per year. The rate of melanoma has nearly tripled in the white population over the past 20 years. ${ }^{1,2}$

Nonmelanoma skin cancer (NMSC) is also on the rise. From 2002 to 2006, there was a 16\% increase in procedures for NMSC. In 2006, the total number of NMSCs in the US population was estimated to be $3,507,693.3$

The risk factors for skin cancers include both environmental and genetic factors. Epidemiologic evidence has shown an increased risk of melanoma among those with extensive exposure to sunlight. ${ }^{4}$ Intermittent intense sun exposure in adolescence, often associated with a sunburn, confers an increased risk of melanoma. ${ }^{5}$ Sites closer to the equator have an increased risk, ${ }^{6}$ and a north-south gradient of melanoma has been shown in the United States. ${ }^{1}$ In contrast, NMSC is associated with cumulative sun exposure risk, rather than the intensity of the exposure, as is seen with melanoma. High numbers of nevi on the body and fair complexion are also associated with increased risk of skin cancer. ${ }^{7}$

Immunosuppressive medications are another important risk factor for skin cancers. Solidorgan transplant recipients have a 3.4-fold increased risk of melanoma ${ }^{8}$; the risk of NMSC in this population is estimated to be even higher. For example, squamous cell skin cancer is 65-250 times more common in posttransplant populations. ${ }^{9}$ In general, the risk of skin cancer correlates with degree of immunosuppression. ${ }^{8}$ Consequently, it is recommended that organ transplant recipients undergo routine skin surveillance due to the increased risk of skin cancer in this population. ${ }^{10}$ Recent data have shown an increased risk of NMSC in patients with inflammatory bowel disease (IBD), ${ }^{11-13}$ particularly associated with the thiopurine class of medications. Little is known about the risk of melanoma in patients with IBD and the specific risks of various immunosuppressive medications used in the treatment of IBD, including the biologic anti-tumor necrosis factor a (anti-TNF) medications. Case reports and a recent meta-analysis have suggested an association between biologic anti-TNF medications and risk of melanoma. ${ }^{14-16}$ Defining the risk of melanoma in patients with IBD is paramount, because preventive measures (such as sunscreen use) have been shown to reduce the incidence of melanoma. ${ }^{17}$

The specific aims of this study were (1) to quantify the risk of melanoma and NMSC among a US cohort of patients with IBD as compared with a non-IBD cohort and (2) to evaluate the risk of melanoma and NMSC among patients with IBD who use immunosuppressive or biologic anti-TNF medications as compared with patients with IBD who have not used these medications. 


\section{Patients and Methods}

We analyzed the inpatient and outpatient procedural and outpatient pharmaceutical insurance claims contained in the LifeLink Health Plan Claims Database (IMS Health, Norwalk, CT) for the period January 1, 1997, through December 31, 2009. The source database contains enrollment information on more than 60 million persons from more than 98 health plans across the United States. This longitudinal, patient-level database has also been used in previous epidemiologic studies of IBD. ${ }^{11,18}$ The specific data extract used for this study includes data covering a long period to better study newer medications used in the treatment of IBD. Prior studies have reported the LifeLink Health Plan Claims Database to be representative of the commercially insured US population on a variety of demographic measures. ${ }^{19}$

\section{Study Design}

We performed a retrospective cohort study to determine the overall risk of melanoma and NMSC in patients with IBD compared with a non-IBD cohort. We then performed nested case-control studies to determine the independent effects of medication use (immunosuppressive and biologic anti-TNF therapy) on melanoma and NMSC among patients with IBD. A similar design has previously been used by our group ${ }^{11}$ and also by Gupta et $\mathrm{al}^{20}$ to evaluate the incidence of disorders in patients with IBD and the effects of various medications.

\section{Cohort Study}

Patient selection-Eligibility criteria for inclusion in the cohort were at least 12 months of continuous health plan enrollment with pharmacy benefits, no history of human immunodeficiency virus (HIV) due to inherent immune system differences, and age younger than 64 years. We chose 64 years as the upper age limit to avoid the possibility of missing data resulting from Medicare dual eligibility (which begins at age 65 years). We identified cases of Crohn's disease (CD) and ulcerative colitis (UC) using a previously reported administrative definition updated to include medications recently approved for IBD indications. ${ }^{21}$ Specifically, patients with IBD were identified by either of 2 definitions: (1) a minimum of 3 health care contacts, on different dates, associated with an International Classification of Diseases, 9th Revision, Clinical Modification (ICD-9) diagnosis code for $\mathrm{CD}$ (555.xx) or UC (556.xx) or (2) at least one claim for CD or UC plus a pharmacy claim for any of the following medications: mesalamine, olsalazine, balsalazide, sulfasalazine, 6mercaptopurine, azathioprine, methotrexate, infliximab, adalimumab, certolizumab pegol, natalizumab, and enteral budesonide. For patients who had claims for both CD and UC, disease assignment was made according to the majority of the last 9 claims or, if there were fewer than 9 claims, the majority of available claims. Those with equal numbers of $\mathrm{CD}$ and UC claims were categorized as unknown IBD type. For the comparison cohort, each patient with IBD was matched to 4 non-IBD persons by age, sex, and month of enrollment ( \pm 6 months). Persons having any IBD claims (based on ICD-9) who did not meet our definition of IBD were ineligible for the comparison cohort. 
Cohort lead time and follow-up-For the entire cohort, we required 6 months of lead time or screening time before the start of follow-up for outcome ascertainment to minimize possible inclusion of prevalent outcomes and to allow assessment of potentially confounding patient characteristics at the time of entry into the cohort. Thus, for patients with IBD, we required 6 months of enrollment before the first qualifying IBD claim; for non-IBD, we required 6 months of enrollment before the enrollment date of the case to whom they were matched.

Each member of the cohort was followed up until the first diagnosis of melanoma or, if none, until censoring at the earlier of one of 2 events: discontinuation of primary or pharmacy insurance coverage or age older than 64 years.

Assessment of melanoma outcome-The primary outcome of interest was first diagnosis of melanoma. The standard definition included both pathologic and surgical components, requiring 2 separate elements within 30 days: (1) a claim containing a Current Procedural Terminology, 4th edition (CPT-4) code for interpretation of pathology from an office or surgical setting (88301-88309) accompanied by an ICD-9 diagnosis code of melanoma (172.0-172.9) on the same line AND (2) within 30 days, a claim containing a CPT-4 code for excision of malignant lesions (11600-11646) or a CPT-4 code for Mohs stage 1 excision (17311 or 17313).

To assess the adequacy of our primary definition, we also specified 2 alternate definitions, one more sensitive and one more specific. The more sensitive definition was any single ICD-9 code for melanoma (172.0-172.9). This definition has been validated within Medicare administrative data via linkage to the Surveillance, Epidemiology, and End Result (SEER) tumor registry and has been found to have a sensitivity of $90.1 \% .^{22}$ The more specific definition required all 3 diagnostic, pathologic, and surgical components (CPT-4 code for pathology AND ICD-9 for melanoma AND CPT-4 for surgery or excision) on the same date. These alternate definitions were used to perform sensitivity analyses of our primary definition of melanoma. Persons with any melanoma claim (ICD-9 172.0-172.9) during the 6-month lead or screening period (before start of follow-up) were excluded to minimize possible inclusion of prevalent cases.

Assessment of NMSC outcome-The definition of NMSC was based on a definition we used in prior work, updated to account for changes in procedure codes for Mohs excision that went into effect in 2007. ${ }^{11}$ The NMSC definition consisted of (1) a claim containing a CPT-4 code for interpretation of pathology from an office or surgical setting (88301-88309 and/or 88329-88332) and an ICD-9 diagnosis code of NMSC (173.0-173.9 or 232.0-232.9) or (2) a claim containing a CPT-4 code for destruction or excision (116xx, 172xx, 1730017315) and one of the previously mentioned ICD-9 codes of NMSC.

Assessment of exposures-Patient exposures and potentially confounding characteristics were assessed using claims from the 6-month lead or screening period before start of follow-up in 3 domains: utilization, comorbidity, and medication use. Health care utilization was defined as the total number of discrete days with at least one nonpharmacologic health care contact over the screening period. Utilization has been 
measured in this fashion in prior pharmacoepidemiology studies of IBD in this data set. ${ }^{23}$ To identify comorbidities, we used the enhanced version of the validated Charlson-Deyo comorbidity index for administrative data developed by Quan et al. ${ }^{24,25}$ This is a score composed of weighted values of 17 selected comorbidities, where higher scores are associated with greater burden of comorbid disease. We excluded malignancy from the list of comorbidities for the melanoma outcome, because melanoma itself is an included malignancy. Use of immunosuppressive or biologic anti-TNF medication use was dichotomized as any use versus none for each of the following medications: 6mercaptopurine, azathioprine, methotrexate, tacrolimus, cyclosporine, mycophenolate mofetil, infliximab, adalimumab, certolizumab pegol, or natalizumab. Any use was defined as one or more claims for a given medication. We also assessed use of 5-aminosalicylic acid (5-ASA) medications (sulfasalazine, balsalazide, mesalamine, olsalazine) in the same any/ none fashion.

Statistical analysis-We used descriptive statistics to summarize characteristics of patients with and without IBD. Continuous variables are reported as mean \pm SD or median with interquartile range, and categorical variables are reported as percentages. We calculated incidence rates of melanoma and NMSC (per 100,000 person-years) and incidence rate ratios (IRRs) comparing the incidence of melanoma and NMSC in IBD and non-IBD patients. To control for confounding, we first assessed possible violation of the proportional hazards assumption via log-log plots and testing of Schoenfeld residuals. Because the assumption was not violated, we used Cox proportional hazard models to calculate hazard ratios (HRs) and 95\% confidence intervals (CIs) for the risk of melanoma and NMSC in the IBD cohort as compared with the non-IBD cohort, controlling for health care utilization and comorbidities. In stratified analyses of $\mathrm{CD}$ and $\mathrm{UC}$, the control group was limited to the respective matched non-CD and matched non-UC subjects.

\section{Nested Case-Control Study}

We next conducted a case-control study evaluating the association between immunosuppressive or biologic anti-TNF medication use and melanoma. This study was nested within the previously defined cohort of patients with IBD. An identical method was used to assess associations between medication use and the NMSC outcome.

Selection of cases and controls-For each case-control study, cases were patients with IBD who were diagnosed with melanoma or NMSC, respectively, and controls were patients with IBD who were disease-free (melanoma or NMSC) at the time of selection. Each case patient was matched to up to 4 IBD patient controls on sex, age (within 2 years), region of the country, disease type (CD or $\mathrm{UC}$ ), and month of the case's diagnosis incidence density sampling. In this sampling technique, potential controls for a given case need only be diseasefree up to the index date, which is the diagnosis date of the case being matched. A control may become a case subsequent to this date, and once a person is diagnosed (becomes a case), he or she is no longer eligible to serve as a control. The index date for each matched set is the date of diagnosis of the case; all exposures were assessed retrospectively from this date. 


\section{Assessment of exposures}

Medication use: The primary medications evaluated included azathioprine and 6mercaptopurine (thiopurine class), methotrexate, tacrolimus and cyclosporine (calcineurin class), mycophenolate mofetil, infliximab, adalimumab, certolizumab pegol (biologic antiTNF class), and natalizumab. We also assessed any 5-ASA medication use (sulfasalazine, balsalazide, mesalamine, olsalazine). All medication use preceding the diagnosis of melanoma or NMSC in cases or preceding the matching index date in controls was analyzed in an any/none fashion.

Other: Health care utilization and comorbidities were determined in the same manner as in the cohort except the referent period for these exposures was the period between 4 and 9 months before the index date for each matched set. The offset of 4 months before diagnosis was chosen so that increased utilization or comorbidities associated with the diagnosis of melanoma or NMSC would not be included in the exposure assessments. As in the cohort, utilization was defined as the number of distinct nonpharmaceutical claim dates in the reference period, and comorbidities were assessed using the Quan update of the Deyo comorbidity index for administrative claims data, excluding malignancies. ${ }^{24,25}$

Statistical analysis-Characteristics of cases and controls were summarized using descriptive statistics. Bivariate analyses were used to compare medication use within cases and controls. We used conditional logistic regression to calculate crude and adjusted odds ratios (ORs) and 95\% CIs for each medication exposure and case status. We controlled for potential confounders, including other medication use, health care utilization, and comorbidities. Analyses were performed for all patients with IBD and also stratified by UC and $\mathrm{CD}$ subtypes.

\section{Results Cohort Study}

The cohort study population included 108,579 patients with IBD. Of these, 50,920 had CD, 56,390 had UC, and 1269 had IBD with unknown type. There were a total of 434,233 individuals in the non-IBD comparison cohort. The median length of follow-up within this cohort was 24 months (interquartile range, 12-43), with a range from 1 to 138 months. Length of follow-up was similar for CD (35 months; interquartile range, 21-52) and UC populations (37 months; interquartile range, 22-57) while significantly less in the non-IBD comparison cohort (21 months; interquartile range, 10-39; $P<.01$ ). Characteristics of the patients with IBD as compared with the non-IBD cohort are shown in Table 1. Characteristics in general were well matched, with increased health care utilization and immunosuppressive medication use among the patients with IBD and among the patients with CD as compared with patients with UC.

Melanoma-For patients with IBD, the overall annual incidence of melanoma was $57.1 / 100,000$, compared with 44.1/100,000 in the non-IBD cohort (Figure 1). When melanoma was evaluated within 10-year age strata, incidence increased with advancing age for both the IBD and non-IBD cohort. Patients with IBD had an increased risk of melanoma 
(IRR, 1.29; 95\% CI, 1.09-1.53). There were differential findings by disease subtype, with significantly increased risk for CD (IRR, 1.45; 95\% CI, 1.13-1.85) but not for UC (IRR, $1.13 ; 95 \%$ CI, 0.89-1.42).

When using Cox proportional hazards modeling to adjust for health care utilization and comorbidities, the risk was not significantly elevated in the overall IBD population as compared with the non-IBD population (adjusted HR, 1.15; 95\% CI, 0.97-1.36). Again, there were differential effects by subtype, with increased risk for CD (adjusted HR, 1.28; 95\% CI, 1.00-1.64) and not for UC (adjusted HR, 1.02; 95\% CI, 0.81-1.29). In analyses using the 2 alternate definitions of the melanoma outcome, one with increased sensitivity and one with increased specificity, the overall IRRs for the IBD population as compared with the non-IBD population were similar (IRR, 1.20 [95\% CI, 0.99-1.44] and IRR, 1.28 [95\% CI, 1.16-1.41], respectively). Table 2 shows the sensitivity analyses (base case, more sensitive, more specific) stratified by disease subtype.

To investigate whether the relative risk of melanoma among patients with IBD is increasing over time, we assessed IRRs in 3 subcohorts defined by date of enrollment in 3 distinct periods (1997-2000, 2001-2004, 2005-2009). In effect, these were 3 separate closed cohorts. In these exploratory analyses, we did find a trend for increasing risk of melanoma with time (IRR of 1.1, 1.3, and 1.5, respectively), providing indirect support for hypotheses that increasing use of immunosuppressive medications may play a causative role. Interestingly, when we also investigated medication use over these periods, biologic antiTNF use dramatically increased. From 1997 to 2000, there were 69 prescriptions of biologic anti-TNF medications per 100,000 person-months. This increased to 2826 per 100,000 person-months from 2005 to 2009. Annual increase in biologic anti-TNF use is shown in Supplementary Figure 1.

NMSC_Patients with IBD had an overall incidence of 912/100,000 for NMSC, as compared with 623/100,000 in the non-IBD comparison cohort. Patients with IBD had an increased risk of NMSC (IRR, 1.46; 95\% CI, 1.40-1.53), as did both CD (IRR, 1.64; 95\% CI, 1.54-1.74) and UC (IRR, 1.34; 95\% CI, 1.26-1.42) subtypes. Similar results were found in analyses adjusted for comorbidities and health care utilization, with an increased risk in the IBD population overall (adjusted HR, 1.34; 95\% CI, 1.28-1.40) and for CD (adjusted HR, 1.48; 95\% CI, 1.39-1.58) and UC (adjusted HR, 1.23; 95\% CI, 1.16-1.31).

\section{Nested Case-Control Study}

Melanoma-The study population for the nested case-control study of melanoma in patients with IBD included 209 cases of melanoma and 823 matched controls ( 2 cases of melanoma could not be matched, and a few cases had <4 matches). Health care utilization, exposure to any biologic anti-TNF medication, and any 5-ASA medication were all higher among cases than controls (Table 3). Differences in any use of 5-ASA, thiopurine, and biologic anti-TNF medication use are shown in Table 4. In the overall study population and in the CD stratum, cases had significantly greater biologic anti-TNF medication use when compared with controls. 
Adjusted multivariate analyses are shown in Table 5. In the overall IBD population, use of any biologic anti-TNF medication was associated with melanoma in crude (OR, 2.08; 95\% CI, 1.24-3.51) and adjusted analyses (OR, 1.88; 95\% CI, 1.08-3.29). There was no significant association with any thiopurine or any 5-ASA use. We performed 2 exploratory subanalyses in an attempt to evaluate whether the risk associated with anti-TNF biologics was associated with length of exposure. In the subset of persons with 1 or more years of enrollment before melanoma (or control index date), there was no risk associated with antiTNF biologic exposure less than 120 days' duration (crude OR, 0.97; 95\% CI, 0.19-4.98). In a second subanalysis, we defined a surrogate marker for long-term use based on current use of anti-TNF biologics at the time of entry into cohort follow-up versus patients who were not using these drugs at enrollment. The adjusted OR for this indicator (long-term vs non-long-term use) was 3.93 (95\% CI, 1.82-8.50).

NMSC - The nested case-control study of patients with IBD who had NMSC included 3288 cases of NMSC and 12,945 controls. Similar to the melanoma case-control study, health care utilization and immunosuppressive medication use (anti-TNF biologic and thiopurine) were higher among cases of NMSC (data not shown). Any thiopurine use was associated with an increased risk of NMSC in crude (OR, 1.98; 95\% CI, 1.79-2.20) and adjusted analyses (adjusted OR, 1.85; 95\% CI, 1.66-2.05). However, anti-TNF biologics were not associated with significantly increased risk in adjusted analyses (OR, 1.14; 95\% CI, 0.951.36). Summary medication effects are shown in Table 5. As with our prior study of NMSC in IBD, we also assessed NMSC risk by medication duration. We investigated both recent (3 months) and persistent use ( $\geq 1$ year). We found increasing risk associated with increasing duration of both anti-TNF biologic and thiopurine medications (data not shown). In a subanalysis, combined use of thiopurines and biologics for $\geq 1$ year was associated with the greatest increased NMSC risk (adjusted OR, 3.89; 95\% CI, 2.33-6.46), followed by persistent thiopurine use alone and biologic anti-TNF use alone as compared with no use of these medications (Supplementary Table 1).

\section{Discussion}

Over the past 2 decades, there have been important advances in the therapy of IBD, including the addition of efficacious medications such as thiopurines and biologic anti-TNF agents to the therapeutic armamentarium. These medications have allowed for dramatic improvements in maintenance of clinical remission, endoscopic remission, and quality of life for patients with IBD. As these medications are used in increasing numbers for individuals with IBD, it becomes important to understand complications that may be associated with these medications, such as malignancy. Some malignancies in patients with IBD, such as skin cancer, are potentially preventable with modification of concomitant risk factors.

The risks of lymphoma, ${ }^{26}$ and more recently NMSC, ${ }^{11-13}$ associated with thiopurine use have been well described. Less is known about the risks of other malignancies, such as melanoma, and the risks of biologic anti-TNF medications. 
Other immunosuppressed populations, such as organ transplant recipients, have a markedly increased risk of NMSC and, to a lesser extent, increased risk of melanoma. In Europe and Australia, the risk of melanoma has been reported to be 1.6-fold to 4-fold higher in transplant recipients when compared with the general population, ${ }^{9}$ where the mean interval between transplantation and diagnosis of melanoma was 5 years. Melanoma accounts for $6.2 \%$ of posttransplantation skin cancers in adults and $15 \%$ in children. ${ }^{9}$

In our study, we found an increased risk of melanoma in patients with CD (adjusted HR, $1.28 ; 95 \% \mathrm{CI}, 1.00-1.64)$. The increased risk may be related to a combination of factors, including the immune dysfunction associated with IBD, the medications used in the therapy of IBD, or increased detection of melanoma in this population related to residual confounding from IBD-associated increased health care utilization. We found an incidence of melanoma in patients with IBD of 57.1/100,000, with a further increased incidence among patients with CD of $60.8 / 100,000$. A prior study of melanoma risk in the US rheumatology population, where similar immune dysfunction and medication therapies exist, found a markedly similar incidence of melanoma of $65.6 / 100,000 .{ }^{27}$ In other populationbased studies of malignancy in IBD, including a recent meta-analysis, the risk of melanoma has not been found to be increased. ${ }^{23-31}$ We speculate that this may be related to the large sample size needed to detect a difference in a rare outcome such as melanoma and because many of these studies were conducted in an era before widespread use of immunosuppressive medications and biologic anti-TNF medications. Indeed, in a subanalysis of time trends, we found that the risk of melanoma in the IBD cohort, relative to the comparison cohort, increased from 1.1 between 1997 and 2000 to 1.5 between 2005 and 2009. Over this same period, prescriptions for anti-TNF biologic agents also dramatically increased. This provides ecological support for the hypothesis that increasing use of antiTNF agents may be associated with increasing risk of melanoma.

Our nested case-control study results also show an association between medication use and melanoma in patients with IBD. Specifically, we found an increased risk of melanoma with biologic anti-TNF use. An increased risk of melanoma associated with biologic anti-TNF use has also been reported in an observational study of the US rheumatology population (OR, 2.3; 95\% CI, 0.9-5.4). Drug-specific risks in this group showed that infliximab was associated with melanoma (OR, 2.6; 95\% CI, 1.0-6.7). ${ }^{27}$ A similar magnitude of increased risk has been found in a recent meta-analysis of cancer risk in patients with rheumatoid arthritis treated with biologic anti-TNF medications (pooled risk estimate for melanoma, $1.79 ; 95 \% \mathrm{CI}, 0.92-2.67) .{ }^{32}$ The mechanism behind this increased risk is not known. It is possible that melanoma occurs after biologic anti-TNF therapy due to unleashing of a previously checked melanoma by the immune system. Alternately, a melanoma could develop de novo after a biologic anti-TNF medication is initiated due to increased photosensitivity or other alternate mechanism. In our study of NMSC, although there was no increased risk with "any" biologic anti-TNF use, we did find increasing risk with increasing duration of use. The risk of NMSC with $\geq 1$ year of biologic anti-TNF therapy was significantly increased (adjusted OR, 1.58; 95\% CI, 1.16-2.14), similar to our prior work.

We did not find a significant association with any use of thiopurines and melanoma, but this risk did trend upward in the UC population. We found a significantly greater risk of NMSC 
in patients with IBD with any thiopurine use (OR, 1.85; 95\% CI, 1.66-2.05), as has been noted previously. ${ }^{1-13}$ The mechanism of increased NMSC risk is believed to be related to selective photosensitivity to UVA light via 6-thioguanine DNA photoproducts. ${ }^{33,34}$ Because thiopurines do not alter the risk of melanoma to the same extent, a different mechanism of increased risk of melanoma is likely. It is interesting that the OR for melanoma associated with any 5-ASA use was also elevated for patients with UC, although not significantly so. Although photosensitivity has been reported as a side effect of 5-ASA medications, particularly sulfasalazine, ${ }^{35}$ these are not immunosuppressive medications and have not previously been associated with risk of skin cancer. Additionally, in our study of NMSC, 5ASA medications were not associated with increased risk. Also, medications such as 5-ASA, thiopurines, or biologics may be markers of increased disease activity, which may correlate with an increased level of immune dysfunction from the underlying IBD and/or increased opportunities for detection due to more regular health care contact.

There are several strengths to this study. Most importantly, the study population was very large (source population of approximately 60 million individuals), which is essential in a study of rare outcomes such as melanoma. We also had broad geographic diversity, with patients included in the study from all regions of the United States. By matching individuals on health plan region, we were able to select controls from similar latitudes in the United States to partially account for ambient sun exposure. By drawing from a large number of health plans of varying size, type, and location, we believe our results to be broadly generalizable to the commercially insured US population. We obtained complete data on all billed prescriptions to completely capture medication exposures, rather than relying on patient recall or clinical charts where prescriptions may have been written but not filled. We were also able to account for health care utilization, because we had access to all health care contacts in the database. Finally, we were able to assess the risk of NMSC in this population, which was shown to be elevated to a similar extent in prior work. ${ }^{11}$ This allows for a comprehensive review of skin cancer risk in IBD and puts the risk of melanoma in perspective, because the absolute risk of NMSC is much higher.

There are also several limitations to this study. Because this is a study using administrative claims data, there is the possibility of misclassification of exposure and outcome related to the lack of clinical detail. To minimize this risk, we used an IBD exposure definition previously used by our group ${ }^{11,18}$ that requires multiple health contacts and/or IBD-related prescriptions. A similar but less specific administrative case definition has been validated by Herrinton et al and was found to have a sensitivity exceeding $90 \%$ and a positive predictive value exceeding $80 \%$ for overall IBD. ${ }^{36} \mathrm{We}$ used an outcome definition of melanoma consisting of multiple contacts, with pathology and excision coding combined with an ICD-9 code for melanoma. We were unable to validate this outcome within our deidentified data. However, in a validation study linking claims data with population-based cancer registry data in a rheumatoid arthritis population, a cancer definition based on diagnosis codes plus cancer-related procedure codes (such as our definition) had a specificity of $99 \%$ and a sensitivity of $80 \%$ for most types of cancer. ${ }^{37}$ Our definition of NMSC has been used in prior work ${ }^{11}$ and similarly consists of diagnosis and procedure-related codes.

Nonetheless, there may be a measurement error associated with outcome measurement, but because this should be nondifferential in the IBD and non-IBD cohorts, it would bias our 
effect estimate toward the null. We additionally used alternate definitions of melanoma in sensitivity analyses and found similar relative risk results with both a more specific and a more sensitive definition. Further support for the validity of the administrative definition of melanoma used in this study is that the incidence in both the IBD and comparison cohorts increased with age, consistent with prior literature.

Another limitation to this study is that the elderly and uninsured were not represented in our population. To the extent that melanoma increases with age, we were unable to address whether the risks may differ in the elderly. In the general population, the incidence of melanoma gradually increases with age for both men and women, with a peak incidence of 1 in 52 for men and 1 in 123 for women aged 70 years and older; comparatively, these figures are 1 in 158 and 1 in 180 for men and women aged $40-59$ years, respectively. ${ }^{38}$ However, the lack of older persons in our study population does not affect the validity of the relative risk measures (IRRs, HRs, ORs) we presented in the context of the population younger than 65 years. Additionally, because of small numbers of melanoma outcomes and medication exposures in the cohort, we were unable to assess the risk of melanoma with combined antiTNF and thiopurine therapy. We were able to assess this risk for the much more common NMSC and found that combined anti-TNF and thiopurine therapy was associated with the highest relative risk (adjusted OR, 3.89; 95\% CI, 2.33-6.46).

A final set of limitations involves the inability to account for exposures not captured in the claims database, including medication use before health plan enrollment, smoking status, socioeconomic status, and race/ethnicity. We were able to obtain data on chronic obstructive pulmonary disease, which has been used as a proxy for smoking status in other studies using administrative data, and this was not significantly associated with the outcome of melanoma. Race could be an unmeasured confounder if it is associated with both the outcome and the exposure. White race is associated with increased risk of melanoma and NMSC. Race may also be associated with IBD and with immunosuppressive medication use. However, several studies suggest that the incidence of IBD among black subjects is approaching that of white subjects. Because our nested case-control study investigated only the IBD population, race would not have been a confounder unless associated with the medication exposures. Among studies that have shown differences in immunosuppressive medication use by race, many cite access to care and socioeconomic factors as a potential cause. ${ }^{39}$ Patients with IBD are known to have higher socioeconomic status, ${ }^{40}$ and this also could be associated with the outcome of melanoma. ${ }^{41}$ However, in our study, all patients are insured and have access to care, thereby minimizing the potential for confounding by socioeconomic factors. Additionally, we did control for health care utilization within our study, a proxy for access to care.

In summary, we found that increased risk of melanoma in patients with IBD is driven by use of anti-TNF biologics, whereas increased risk of NMSC is driven by use of thiopurines. Although we found an association between biologic anti-TNF use and melanoma, the absolute risk of melanoma in patients with IBD remains quite low at 57/100,000 personyears. By comparison, the absolute risk of NMSC was much greater at 912/100,000 personyears. The mildly increased relative risk of melanoma with biologic anti-TNF medications likely does not outweigh the benefits in patients with moderate to severe IBD and should not 
deter use of these efficacious medications, and neither should the increased relative risk of NMSC with thiopurines. Instead, providers should emphasize the importance of skin cancer prevention in those who require these medications. Options for primary prevention of both melanoma and NSMC include sun avoidance, sun protection via sunscreen, or use of sunprotective clothing. ${ }^{42}$ In fact, a recent randomized controlled trial of sunscreen use in the general population in Australia found that sunscreen reduced the incidence of melanoma. ${ }^{17}$ This has long been known to be true for NMSC. Another consideration for at-risk populations, such as those with IBD, is application of secondary preventive measures such as routine skin screening examinations. This may be particularly important in the IBD population using immunosuppressive medications and warrants further study.

\section{Supplementary Material}

Refer to Web version on PubMed Central for supplementary material.

\section{Acknowledgments}

The statements, findings, conclusions, views, and opinions contained and expressed in this article are based in part on data obtained under license from the following IMS Health Incorporated information service(s): IMS LifeLink Health Plan Claims Database (1997-2009), IMS Health Incorporated. All Rights Reserved. The statements, findings, conclusions, views, and opinions contained and expressed herein are not necessarily those of IMS Health Incorporated or any of its affiliated or subsidiary entities.

Funding

Supported by a Career Development Award from the Crohn's and Colitis Foundation of America (M.D.L. and C.A.P.) and grants K08 DK088957-01 (to M.D.K.) and P30 DK034987 (to R.S.S.) from the National Institutes of Health.

\section{Abbreviations used in this paper}

5-ASA 5-aminosalicylic acid

anti-TNF anti-tumor necrosis factor a

CI confidence interval

CPT-4 Current Procedural Terminology, 4th edition

HR hazard ratio

ICD-9 International Classification of Diseases, 9th Revision, Clinical Modification

IRR incidence rate ratio

NMSC nonmelanoma skin cancer

OR odds ratio

\section{References}

1. National Cancer Institute. SEER stat fact sheets: melanoma of the. skin. Available at: http:// www.seer.cancer.gov/statfacts/html/melan.html\#prevalence

2. Jemal A, Siegel R, Xu J, et al. Cancer statistics, 2010. CA Cancer J Clin. 2010; 60:277-300. [PubMed: 20610543] 
3. Rogers HW, Weinstock MA, Harris AR, et al. Incidence estimate of nonmelanoma skin cancer in the United States, 2006. Arch Dermatol. 2010; 146:283-287. [PubMed: 20231499]

4. Gilchrest BA, Eller MS, Geller AC, et al. The pathogenesis of melanoma induced by ultraviolet radiation. N Engl J Med. 1999; 340:1341-1348. [PubMed: 10219070]

5. Elwood JM, Jopson J. Melanoma and sun exposure: an overview of published studies. Int J Cancer. 1997; 73:198-203. [PubMed: 9335442]

6. Bulliard JL, Cox B, Elwood JM. Latitude gradients in melanoma incidence and mortality in the nonMaori population of New Zealand. Cancer Causes Control. 1994; 5:234-240. [PubMed: 8061171]

7. Chaudru V, Chompret A, Bressac-de Paillerets B, et al. Influence of genes, nevi, and sun sensitivity on melanoma risk in a family sample unselected by family history and in melanoma-prone families. J Natl Cancer Inst. 2004; 96:785-795. [PubMed: 15150307]

8. Jensen P, Hansen S, Moller B, et al. Skin cancer in kidney and heart transplant recipients and different long-term immunosuppressive therapy regimens. J Am Acad Dermatol. 1999; 40:177-186. [PubMed: 10025742]

9. Euvrard S, Kanitakis J, Claudy A. Skin cancers after organ transplantation. N Engl J Med. 2003; 348:1681-1691. [PubMed: 12711744]

10. Berg D, Otley CC. Skin cancer in organ transplant recipients: Epidemiology, pathogenesis, and management. J Am Acad Dermatol. 2002; 47:1-17. quiz 18-20. [PubMed: 12077575]

11. Long MD, Herfarth HH, Pipkin CA, et al. Increased risk for nonmelanoma skin cancer in patients with inflammatory bowel disease. Clin Gastroenterol Hepatol. 2010; 8:268-274. [PubMed: 20005977]

12. Peyrin-Biroulet L, Khosrotehrani K, et al. Increased risk for nonmelanoma skin cancers in patients who receive thiopurines for inflammatory bowel disease. Gastroenterology. 2011; 141:1621-1628. e1-5. [PubMed: 21708105]

13. Singh H, Nugent Z, Demers AA, et al. Increased risk of nonmelanoma skin cancers among individuals with inflammatory bowel disease. Gastroenterology. 2011; 141:1612-1620. [PubMed: 21806945]

14. Kowalzick L, Eickenscheidt L, Komar M, et al. [Long term treatment of psoriasis with TNF-alpha antagonists Occurrence of malignant melanoma.]. Hautarzt. 2009; 60:655-657. [PubMed: 19280165]

15. Fulchiero GJ Jr, Salvaggio H, Drabick JJ, et al. Eruptive latent metastatic melanomas after initiation of antitumor necrosis factor therapies. J Am Acad Dermatol. 2007; 56:S65-S67. [PubMed: 17434043]

16. Khan I, Rahman L, McKenna DB. Primary cutaneous melanoma: a complication of infliximab treatment? Clin Exp Dermatol. 2009; 34:524-526. [PubMed: 19196307]

17. Bigby M, Kim CC. A prospective randomized controlled trial indicates that sunscreen use reduced the risk of developing melanoma. Arch Dermatol. 2011; 147:853-854. [PubMed: 21768486]

18. Long MD, Porter CQ, Sandler RS, et al. Suboptimal rates of cervical testing among women with inflammatory bowel disease. Clin Gastroenterol Hepatol. 2009; 7:549-553. [PubMed: 18996498]

19. Stempel DA, Mauskopf J, McLaughlin T, et al. Comparison of asthma costs in patients starting fluticasone propionate compared to patients starting montelukast. Respir Med. 2001; 95:227-234. [PubMed: 11266241]

20. Gupta G, Lautenbach E, Lewis JD. Incidence and risk factors for herpes zoster among patients with inflammatory bowel disease. Clin Gastroenterol Hepatol. 2006; 4:1483-1490. [PubMed: 17162240]

21. Kappelman MD, Rifas-Shiman SL, Kleinman K, et al. The prevalence and geographic distribution of Crohn's disease and ulcerative colitis in the United States. Clin Gastroenterol Hepatol. 2007; 5:1424-1429. [PubMed: 17904915]

22. Barzilai DA, Koroukian SM, Neuhauser D, et al. The sensitivity of Medicare data for identifying incident cases of invasive melanoma (United States). Cancer Causes Control. 2004; 15:179-184. [PubMed: 15017130]

23. Lund JL, Sturmer T, Porter CQ, et al. Thiazolidinedione use and ulcerative colitis-related flares: an exploratory analysis of administrative data. Inflamm Bowel Dis. 2011; 17:787-794. [PubMed: 20848530] 
24. Deyo RA, Cherkin DC, Ciol MA. Adapting a clinical comorbidity index for use with ICD-9-CM administrative databases. J Clin Epidemiol. 1992; 45:613-619. [PubMed: 1607900]

25. Quan H, Sundararajan V, Halfon P, et al. Coding algorithms for defining comorbidities in ICD-9CM and ICD-10 administrative data. Med Care. 2005; 43:1130-1139. [PubMed: 16224307]

26. Siegel CA, Marden SM, Persing SM, et al. Risk of lymphoma associated with combination antitumor necrosis factor and immunomodulator therapy for the treatment of Crohn's disease: a metaanalysis. Clin Gastroenterol Hepatol. 2009; 7:874-881. [PubMed: 19558997]

27. Wolfe F, Michaud K. Biologic treatment of rheumatoid arthritis and the risk of malignancy: analyses from a large US observational study. Arthritis Rheum. 2007; 56:2886-2895. [PubMed: 17729297]

28. Pedersen N, Duricova D, Elkjaer M, et al. Risk of extra-intestinal cancer in inflammatory bowel disease: meta-analysis of population-based cohort studies. Am J Gastroenterol. 2010; 105:1480 1487. [PubMed: 20332773]

29. Bernstein CN, Blanchard JF, Kliewer E, et al. Cancer risk in patients with inflammatory bowel disease: a population-based study. Cancer. 2001; 91:854-862. [PubMed: 11241255]

30. Ekbom A, Helmick C, Zack M, et al. The epidemiology of inflammatory bowel disease: a large, population-based study in Sweden. Gastroenterology. 1991; 100:350-358. [PubMed: 1985033]

31. Katsanos KH, Vermeire S, Christodoulou DK, et al. Dysplasia and cancer in inflammatory bowel disease 10 years after diagnosis: results of a population-based European collaborative follow-up study. Digestion. 2007; 75:113-121. [PubMed: 17598963]

32. Mariette X, Matucci-Cerinic M, Pavelka K, et al. Malignancies associated with tumour necrosis factor inhibitors in registries and prospective observational studies: a systematic review and metaanalysis. Ann Rheum Dis. 2011; 70:1895-1904. [PubMed: 21885875]

33. O'Donovan P, Perrett CM, Zhang X, et al. Azathioprine and UVA light generate mutagenic oxidative DNA damage. Science. 2005; 309:1871-1874. [PubMed: 16166520]

34. Parrish JA. Immunosuppression, skin cancer, and ultraviolet A radiation. N Engl J Med. 2005; 353:2712-2713. [PubMed: 16371639]

35. Horiuchi Y, Shimakura S. Mesalazine and photosensitivity. Am J Gastroenterol. 1999; 94:33863387. [PubMed: 10566765]

36. Herrinton LJ, Liu L, Lafata JE, et al. Estimation of the period prevalence of inflammatory bowel disease among nine health plans using computerized diagnoses and outpatient pharmacy dispensings. Inflamm Bowel Dis. 2007; 13:451-461. [PubMed: 17219403]

37. Setoguchi S, Solomon DH, Weinblatt ME, et al. Tumor necrosis factor alpha antagonist use and cancer in patients with rheumatoid arthritis. Arthritis Rheum. 2006; 54:2757-2764. [PubMed: 16947774]

38. American Cancer Society. Cancer facts \& figures 2012. Atlanta, GA: American Cancer Society; 2012.

39. Flasar MH, Johnson T, Roghmann MC, et al. Disparities in the use of immunomodulators and biologics for the treatment of inflammatory bowel disease: a retrospective cohort study. Inflamm Bowel Dis. 2008; 14:13-19. [PubMed: 17973305]

40. Green C, Elliott L, Beaudoin C, et al. A population-based ecologic study of inflammatory bowel disease: searching for etiologic clues. Am J Epidemiol. 2006; 164:615-623. discussion 624-628. [PubMed: 16920784]

41. Singh SD, Ajani UA, Johnson CJ, et al. Association of cutaneous melanoma incidence with areabased socioeconomic indicators-United States, 2004-2006. J Am Acad Dermatol. 2011; 65:S58S68. [PubMed: 22018068]

42. Long MD, Kappelman MD, Pipkin CA. Nonmelanoma skin cancer in inflammatory bowel disease: a review. Inflamm Bowel Dis. 2011; 17:1423-1427. [PubMed: 21053358] 


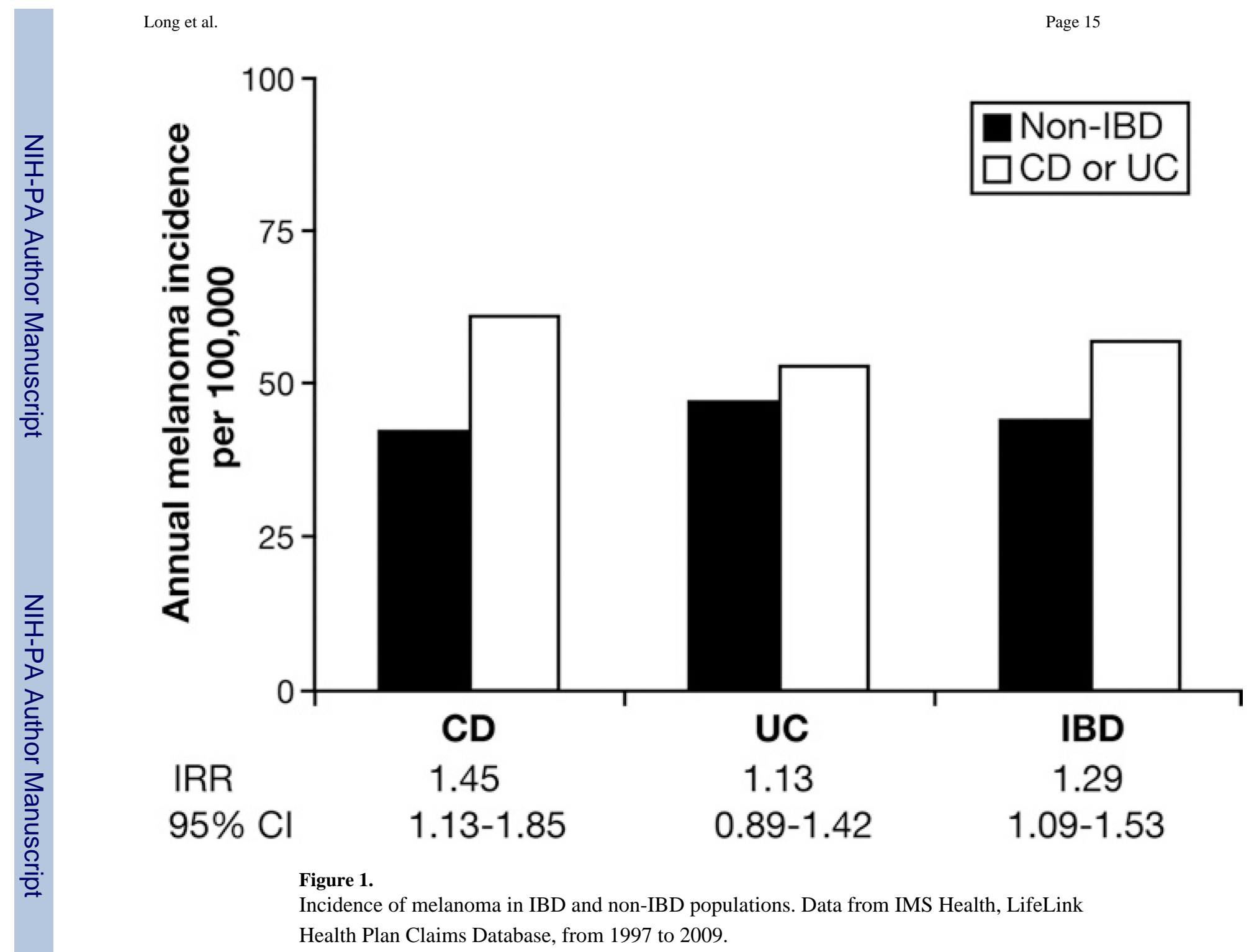




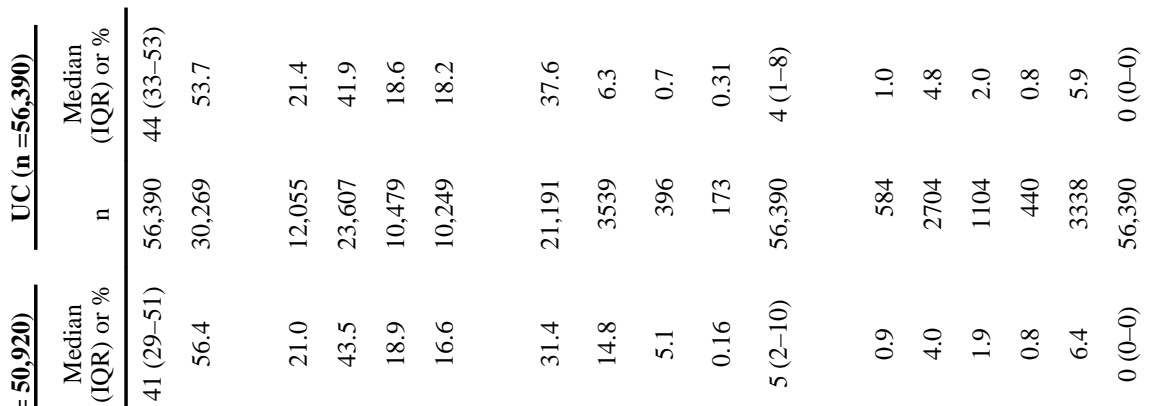

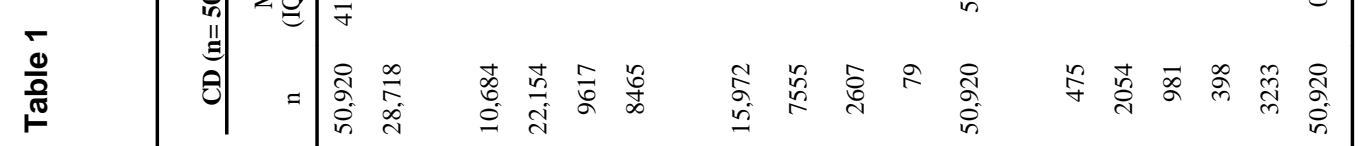

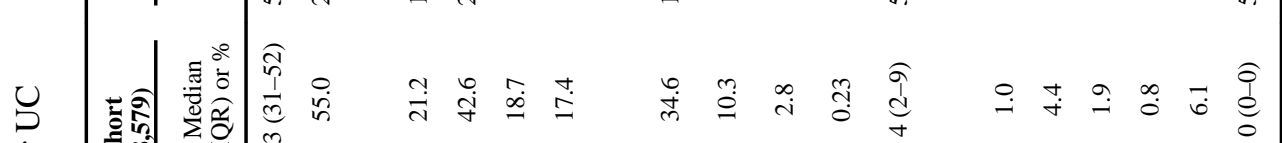




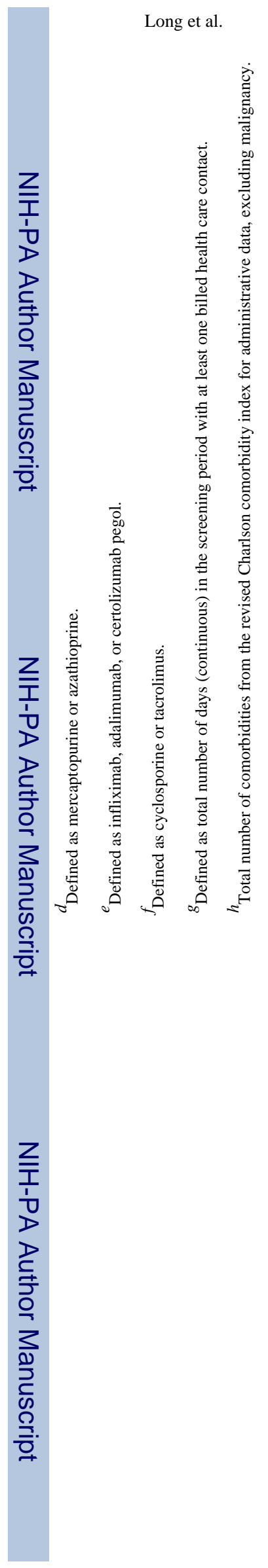

Page 17

Gastroenterology. Author manuscript; available in PMC 2014 June 21. 
Table 2

Sensitivity Analyses of Melanoma, Overall and Stratified By CD or UC, as Compared With the Non-IBD Cohort

\begin{tabular}{lccc}
\hline & $\begin{array}{c}\text { IBD overall } \\
(\mathbf{n}=\mathbf{1 0 8 , 5 1 8}) \\
\text { IRR }(\mathbf{9 5 \%} \mathbf{C I})\end{array}$ & $\begin{array}{c}\text { CD } \\
(\mathbf{n}=\mathbf{5 0 , 9 2 0}) \\
\text { IRR }(\mathbf{9 5 \%} \mathbf{C I})\end{array}$ & $\begin{array}{c}\text { UC } \\
(\mathbf{n}=\mathbf{5 6 , 3 9 0}) \\
\text { IRR (95\% CI) }\end{array}$ \\
\hline Sensitivity analyses & & & \\
Base case $^{a}$ & $1.29(1.09-1.53)$ & $1.45(1.13-1.85)$ & $1.13(0.89-1.42)$ \\
More sensitive $^{b}$ & $1.28(1.16-1.41)$ & $1.31(1.14-1.52)$ & $1.24(1.08-1.41)$ \\
More specific $^{c}$ & $1.20(0.99-1.44)$ & $1.30(0.98-1.73)$ & $1.10(0.85-1.41)$ \\
\hline
\end{tabular}

NOTE. Matched characteristics include age (within 2 years), sex, region of the country, and IBD subtype. Data from IMS Health, LifeLink Health Plan Claims Database, from 1997 to 2009.

${ }^{a}$ Main outcome, a claim containing (1) a CPT-4 code for interpretation of pathology from an office or surgical setting (88301-88309) accompanied by an ICD-9 diagnosis code of melanoma (172.0-172.9) on the same line AND (2) within 30 days, a claim containing a CPT-4 code for excision of malignant lesions (11600-11646) or a CPT-4 code for Mohs stage 1 excision (17311 or 17313).

${ }^{b}$ Any one ICD-9 diagnosis code of melanoma (172.0-172.9).

${ }^{c}$ All 3 diagnostic, pathologic, and surgical components (CPT-4 code for pathology AND ICD-9 for melanoma AND CPT-4 for surgery or excision) on the same date. 


\section{Table 3}

Characteristics of the IBD Population by Melanoma

\begin{tabular}{|c|c|c|c|c|c|}
\hline \multirow[b]{2}{*}{ Characteristics $^{a}$} & \multicolumn{2}{|c|}{ No melanoma $(n=823)$} & \multicolumn{2}{|c|}{ Melanoma $(n=209)$} & \multirow[b]{2}{*}{$P$ value $^{b}$} \\
\hline & $\mathbf{n}$ & $\%$ or median (IQR) & $\mathbf{n}$ & \% or median (IQR) & \\
\hline Age $(y)^{c}$ & 823 & $50(41-56)$ & 209 & $50(42-56)$ & .52 \\
\hline Sex $(\%$ female $)$ & 436 & 53.0 & 110 & 52.6 & .93 \\
\hline \multicolumn{6}{|l|}{ IBD type } \\
\hline $\mathrm{CD}$ & 402 & 48.9 & 102 & 48.8 & .47 \\
\hline $\mathrm{UC}$ & 416 & 50.6 & 104 & 49.8 & \\
\hline Unknown & 5 & 0.6 & 3 & 1.44 & \\
\hline Utilization $^{d}$ & 823 & $2(0-5)$ & 209 & $4(1-7)$ & $<.01$ \\
\hline \multicolumn{6}{|l|}{ Region } \\
\hline East & 140 & 17.0 & 36 & 17.2 & .99 \\
\hline Midwest & 368 & 44.7 & 92 & 44.0 & \\
\hline South & 149 & 18.1 & 39 & 18.7 & \\
\hline West & 166 & 20.2 & 42 & 20.1 & \\
\hline \multicolumn{6}{|l|}{ Comorbidities } \\
\hline Cardiac & 10 & 1.2 & 4 & 1.9 & .44 \\
\hline Diabetes mellitus & 51 & 6.2 & 8 & 3.8 & .19 \\
\hline Liver disease & 10 & 1.2 & 3 & 1.4 & .80 \\
\hline Renal & 3 & 0.4 & 3 & 1.4 & .07 \\
\hline Chronic obstructive pulmonary disease & 36 & 4.4 & 12 & 5.7 & .40 \\
\hline No. of comorbidities $e$ & 823 & $0(0-0)$ & 209 & $0(0-0)$ & .71 \\
\hline
\end{tabular}

NOTE. Data from IMS Health, LifeLink Health Plan Claims Database, from 1997 to 2009.

${ }^{a}$ Matched factors include age (within 2 years), sex, health plan region, and IBD subtype.

${ }^{b} \mathrm{P}$ value by Pearson $\chi^{2}$ test for categorical variables and Wilcoxon rank sum test for continuous variables.

${ }^{c}$ Age at time of melanoma (case) or index date (control).

${ }^{d}$ Defined as total number of days (continuous) with any health care contact over a 6-month period.

${ }^{e}$ Number of comorbidities from Charlson comorbidity index, excluding malignancy. 


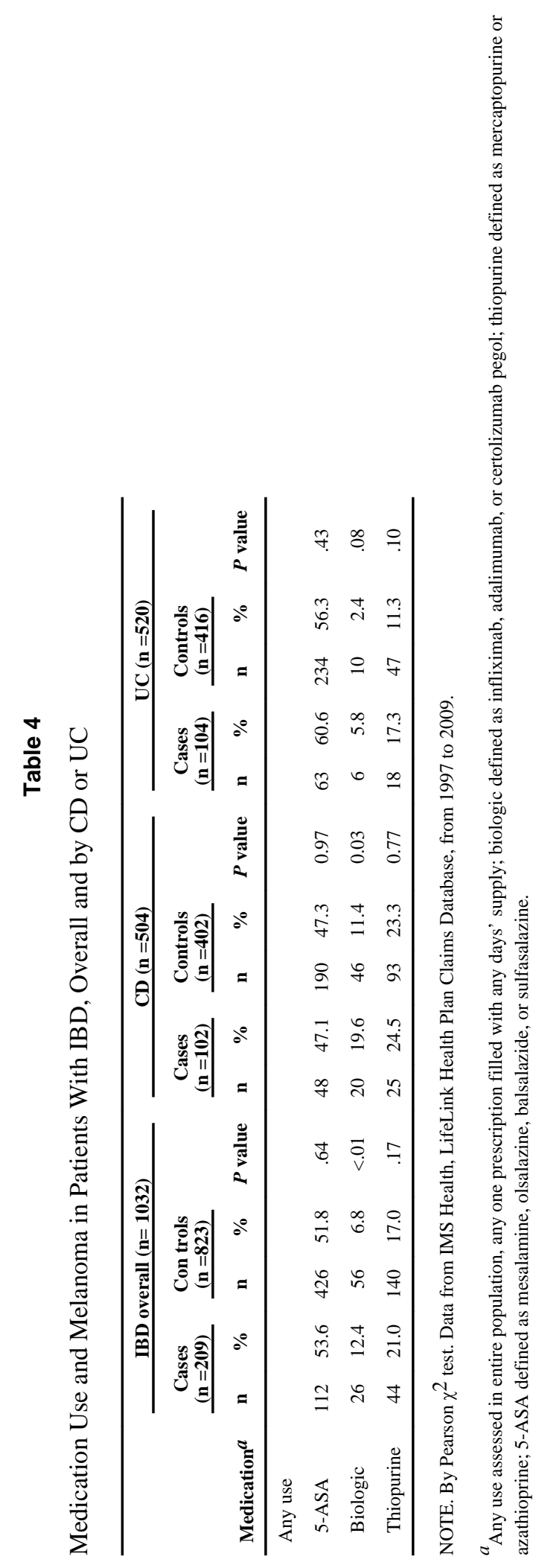




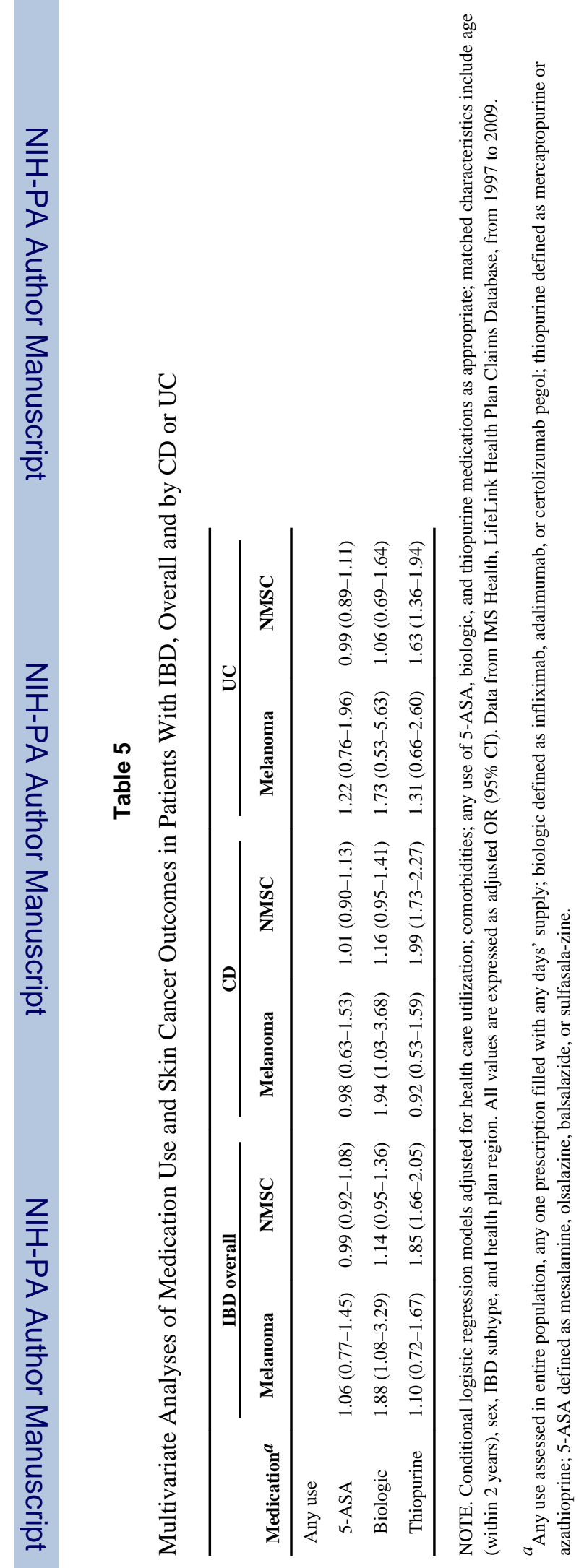

